\title{
Microbial Diversity of Rhizosphere Soil of Tobacco at Different Growth Periods
}

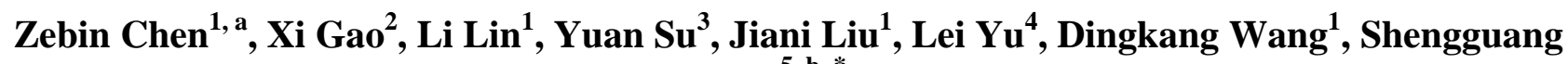 \\ $\mathrm{Xu}^{5, \mathrm{~b}, *}$ \\ ${ }^{1}$ College of Agronomy, Kunming University, Kunming, Yunnan Province, China. \\ ${ }^{2}$ College of Plant Protection, Yunnan Agricultural University, Kunming, Yunnan Province, China. \\ ${ }^{3}$ Key Laboratory of Characteristic Biological Resource Development and Utilization of Colleges and \\ Universities in Yunnan Province, Kunming, Yunnan Province, China. \\ ${ }^{4}$ Yunnan Urban Characteristic Agriculture Engineering Technology Research Center, Kunming, Yunnan \\ Province, China. \\ ${ }^{5}$ Yunnan Institute of Biological Carbon Engineering Research Center, Kunming, Yunnan Province, China. \\ azbchenkmu@163.com, bsgxu2011@126.com \\ *Corresponding author
}

Keywords: high throughput sequencing; growth period; tobacco; rhizosphere; soil microorganism.

Abstract: This paper aims to analyze microbial diversity of tobacco rhizosphere soil at different growth stages. With tobacco rhizosphere soil as the research object, high throughout sequencing was carried out on DNA of 16S rDNA-V4 region and fungi of ITS1 region in rhizosphere soil of tobacco. Soil samples were collected at different growth stages. Qiime and Mothur software were used to collate and count Operational Taxonomic Unit (OTU) in samples; the abundances and Alpha diversities of species were analyzed. The results showed that the order of bacterial diversity was squaring period $>$ decaying period $>$ resettling stage $>$ mature period $>$ vigorous growing period. The diversity of fungi was highest at the mature period, and showed declining trend. At the level of genus, the most dominant bacteria at resettling stage were Pseudomonas, accounting for $2.70 \%$; the most dominant bacteria at vigorous growing period were Alcanivorax, accounting for $15.55 \%$; the most dominant bacteria at mature period were Candidatus Nitrososphaera, accounting for $20.16 \%$; the most dominant bacteria at decaying period were Streptomyces, accounting for $5.16 \%$; the most dominant bacteria at squaring period were Marinobacter, accounting for $14.24 \%$. The diversity analysis showed that dominant bacteria in rhizosphere soil varied greatly at different growth stages. The diversities and abundances of bacteria at the mature, vigorous growing and squaring periods were higher than those of resettling and decaying periods. The most dominant fungi at resettling period were Aspergillus, accounting for $12.19 \%$; the most dominant fungi at vigorous growing period were Mortierella, accounting for 4.29\%; the most dominant fungi at mature period were Un--s-Fungi sp, accounting for 2.91\%; the most dominant fungi at decaying period were Chaetomium, accounting for 3.87\%; the most dominant fungi at squaring period were Un--s-Tremellomycetes sp, accounting for $10.24 \%$. The diversity analysis showed that dominant fungi in rhizosphere soil varied greatly at different growth stages. The species diversities and total abundances of fungi at the resettling period were higher than those of squaring, decaying, mature and vigorous growing periods. Fungal diversity also declined with the passage of time.

\section{Introduction}

Tobacco is one of the most important economic crops widely cultivated in China; China has the largest growing area and the highest total output of tobacco in the world. [1, 2] At present, many scholars believe that the main obstacle of continuous cropping tobacco is the change of rhizosphere micro-ecological environment. With the increase of tobacco cropping years, the micro-ecological balance in rhizosphere is broken; the growth of tobacco is affected. Soil microorganism is an 
important component of micro-ecology in the rhizosphere of plant. It is consisted of three major groups: bacteria, fungi and actinomycetes. It also includes other microorganism groups like ammonifying bacteria, nitrifying bacteria, denitrifying bacteria, aerobic nitrogen fixing bacteria, anaerobic nitrogen fixing bacteria, cellulose decomposing bacteria, phosphorus bacteria and silicate bacteria. Soil microorganisms produce important influences on tobacco. They decompose organic matters to form humus and release nutrients, and transform soil carbon as well as fixed inorganic nutrient elements.[3] Research on the composition and quantity of tobacco rhizosphere microorganisms at different growth stages can provide theoretical basis for controlling soil-borne diseases. Yu-zhen Fan studied changes of Trifolium repens rhizosphere microorganism at different growth stages, and found that Trifolium repens at different growth stages produce similar influences on rhizosphere bacteria, fungi and the total number of microbes; the distribution quantity and rhizosphere effect increased and then decreased with the growing and decaying of the plant, while the rhizosphere actinomycetes decreased and then increased with the growing and decaying of the plant. [4]Jian-bo Sun studied the diversity and quantity of bacteria in the rhizosphere soil of bananas at different growth stages, and found that during the experiment, some bacteria species multiplied with the growing of bananas, leaded to unbalanced soil rhizosphere bacterial community structure and deteriorated soil microbial environment. [5] Qing-hua Wu studied changes in microbes of Aconitum rhizosphere soil at different growth stages, and found that at different growth periods and under different growth conditions, there were significant differences in the bacteria of Aconitum soil; with the growing of plants, diseased and infected plants decreased gradually, while healthy plants decreased first and then increased. [6] At present, study about the microbial composition of rhizosphere soil at different growth stages of tobacco has not been reported. Tobacco is an important economic crop widely cultivated in China. It is necessary to study the diversity of soil microbes of tobacco rhizosphere at different growth stages. In this study, the microbial diversity of tobacco rhizosphere soil at different growth stages was studied by high throughput sequencing technology. The data obtained were more comprehensive and accurate than the first generation sequencing technology. The results could provide scientific basis for the remediation of soil ecological environment.

\title{
2. Research Materials and Methods
}

\subsection{Sample collection.}

The tested tobacco variety was Yunyan 87. In 2016, samples of tobacco rhizosphere soil at the squaring period, decaying period, resettling stage, mature period and vigorous growing period were taken from Xuanwei test field located at Qujing, Yunnan Province. "S" sampling method and hard soil sampling device were used to collect the rhizosphere soil of tobacco. [7] 5 sampling points were selected. $1 \mathrm{~kg}$ soil sample was taken from each point at the depth of $0-20 \mathrm{~cm}$. After uniform mixing at the site, the four division method was used to discard superfluous soil samples. Then the samples were put into the polyethylene bags and sealed. Labels were attached inside and outside the bags, indicating the sampling number, name, depth, location, date and gatherer. Then the samples were taken back to the laboratory for low temperature preservation.

\subsection{Extraction of total DNA.}

Soil DNA was extracted according to the instruction of the soil genome DNA extraction kit (DP336) produced by Tiangen Company

\subsection{PCR amplification.}

PCR was carried out through specific primers of 16S-V4 and ITS1 regions [8].

\subsection{High throughput sequencing of PCR products.}

\author{
Sequencing was commissioned to Beijing Novogene Corporation.
}




\subsection{Bioinformatics analysis.}

Uparse (version 7.1, http://qiime.org/) software platform was used in OTU clustering. RDP classifier bayesian algorithm was employed in the taxonomic analysis of OTU representative sequences at the similarity level of $97 \%$, and in the statistics of sample community composition at different levels. Mothur software was adopted in dilution curve analysis. Shannon index, Simpson index and species richness index (ACE) were used to calculate bacterial ecological diversity index. Thermal image was drawn through Fasttree software and R language. SVG software independently developed by Novogene Corporation was used to draw classification tree graphs of specific species. $[9,10]$

\section{Research Results and Analysis}

\subsection{Analysis of OTU abundance and $\alpha$ diversity index of bacteria and fungi.}

Through sequencing the 16S rDNA-V4 region, the Shannon indexes and Chao1 indexes of rhizosphere soil bacteria at each growth periods were obtained. The sequence of bacterial diversity was: squaring period $>$ decaying period $>$ resettling stage $>$ mature period $>$ vigorous growing period. By sequencing the ITS1 region, it was found that the Shannon indexes of fungi in rhizosphere soil were between 5.5 and 5.9; the value of mature period was the highest, 5.9; the value of resettling stage was the lowest, 5.5. Chao1 indexes were between 416.8 and 696.5. The value of squaring period was the highest, 696.5; the value of decaying period was the lowest, 416.8. The Shannon indexes of fungi in soil samples at the squaring period, decaying period, resettling stage and vigorous growing period were all lower than that of the mature period, and the fungal diversity of the 5 periods increased first and then decreased.

\subsection{Analysis on bacteria and fungi species and abundances.}

At the five growth periods, dominant bacteria were distributed in 4 phyla, namely

Proteobacteria, which accounted for $71.72 \%$, Crenarchaeota, which accounted for $20.16 \%$, Actinobacteria, which accounted for $5.16 \%$ and Bacteroidetes, which accounted for $2.96 \%$. In the phylum of Proteobacteria, the class of $\gamma$ Proteobacteria accounted for the largest proportion of $34.21 \%$; it was followed by $\alpha$ - Proteobacteria class, which accounted for $33.15 \%$, Thaumarchaeota class, which accounted for 20.16\%; Actinobacteria class, which accounted for 5.16\% ; $\beta$ Proteobacteria class, which accounted for $4.36 \%$ and Flavobacteriia class, which accounted for 2.96\%. $\gamma$ Proteobacteria class mainly included following orders: Oceanospirillales, accounting for 15.55\%; Alteromonadales, accounting for 14.24\%; Enterobacteriales, accounting for $1.71 \%$; Oceanospirillales, accounting for $2.70 \%$. The dominant bacteria of rhizosphere soil were the same at vigorous growing period, mature period and squaring period, but the proportions of Alcanivorax, Candidatus Nitrososphaera and Marinobacter were quite different. The proportion of Alcanivorax in the rhizosphere soil at vigorous growing period was obviously higher than that of mature period and squaring period; Candidatus Nitrososphaera in the rhizosphere soil at mature period was obviously higher than that of vigorous growing period and squaring period; the proportion of Marinobacter in soil samples at squaring periodwas significantly higher than that in vigorous growing period and mature period. The dominant bacteria of rhizosphere soil were the same at resettling stage and decaying period, but the proportions of Pseudomonas and Streptomyces were quite different. The proportion of Pseudomonas in the rhizosphere soil at resettling stage was significantly higher than that of decaying period, while the proportion of Streptomyces in the rhizosphere soil at decaying period was significantly higher than that of resettling stage. The proportion of Janthinobacterium in the rhizosphere soil at vigorous growing period was significantly higher than that of squaring and mature periods. The proportion of Bizionia in the rhizosphere soil at the mature period was significantly higher than that of squaring and vigorous growing periods. Candidatus Nitrososphaera, Kaistobacter, Rhodoplanes and Streptomyces could be found in all the five periods. Some bacterial genera only existed in a few periods. For instance Alcanivorax, Marinobacter and Bizionia could be 
found in the samples of squaring period, mature period and vigorous growing period, but could not be found in samples of decaying period and resettling stage.

The dominant fungi in the 5 growth periods were Ascomycota, accounting for $79.23 \%$, Basidiomycota, accounting for $10.24 \%$, Zygomycota, accounting for $7.62 \%$ and Un--s-Fungi SP, accounting for $2.91 \%$. In the phylum of Ascomycota, the class of Sordariomycetes takes the largest proportion of $41.74 \%$, followed by Eurotiomycetes, accounting for $19.12 \%$, Tremellomycetes, accounting for $10.24 \%$, and Dothideomycetes, accounting for $4.49 \%$. The dominant fungi were the same in squaring period, decaying period and resettling stage; but the proportions of Aspergillus, Chaetomium and Un--s-Tremellomycetes sp were significantly different. The proportion of Aspergillus in the rhizosphere soil at resettling stage was obviously higher than that of squaring and decaying periods; the proportion of Chaetomium at the decaying period was significantly higher than that of squaring period and resettling stage; the proportion of Un--s-Tremellomycetes sp at the squaring period was obviously higher than that of decaying period and resettling stage. The dominant fungi at mature and vigorous growing periods were the same. Aspergillus, Un--s-Tremellomycetes sp, Corynascella, Chaetomium, Mortierella, Retroconis, Un--s-Fungi sp, Un--s-Sordariales sp and Un--s-Chaetomiaceae sp could be found in all five periods.

\section{Discussion and Conclusion}

From the relative abundance of various bacteria at different periods, it could be seen that dominant bacteria in the resettling stage were Pseudomonas, Escherichia, Candidatus Nitrososphaera, Streptomyces, Kaistobacter, Rhodoplanes and Janthinobacterium. The abundance of Pseudomonas in the rhizosphere soil at resettling stage was significantly higher than that of decaying period, squaring period, mature period and vigorous growing period. Dominant bacteria in the vigorous growing period were Alcanivorax, Marinobacter, Janthinobacterium, Bizionia, Kaistobacter, Rhodoplanes, Streptomyces, Pseudomonas, Candidatus Nitrososphaera and Escherichia. The abundance of Alcanivorax at the vigorous growing period was significantly higher than that of decaying period, squaring period, mature period and resettling stage. Dominant bacteria in the mature period were Candidatus Nitrososphaera, Alcanivorax, Bizionia, Marinobacter, Rhodoplanes, Kaistobacter, Janthinobacterium, Streptomyces, Pseudomonas and Escherichia. The abundance of Candidatus Nitrososphaera at mature period was significantly higher than that of decaying period, squaring period, vigorous growing period and resettling stage. Dominant bacteria in the decaying period were Streptomyces, Janthinobacterium, Rhodoplanes, Candidatus Nitrososphaera, Kaistobacter, Escherichia and Pseudomonas.

Dominant fungi at the resettling stage were Aspergillus, Corynascella, Retroconis, Chaetomium, Chrysosporium, Un--s-Chaetomiaceae sp, Un--s-Sordariales sp, Penicillium, Pulvinula and Un--s-Fungi sp. The abundance of Aspergillus at resettling stage was significantly higher than that of decaying period, squaring period, vigorous growing period and mature stage. Dominant fungi at the vigorous growing period were Mortierella, Aspergillus, Chaetomium, Corynascella, Retroconis, Un--s-Chaetomiaceae sp, Un--s-Sordariales sp, Un--s-Tremellomycetes sp, Mortierella and Tomentella. The abundance of Mortierella at the vigorous growing period was significantly higher than that of decaying period, squaring period, mature period and resettling stage. Dominant fungi at the mature period were Un--s-Fungi sp, Aspergillus, Corynascella, Chaetomium, Un--s-Tremellomycetes sp, Un--s-Sordariales sp, Un--s-Chaetomiaceae sp, Retroconis, Mortierella, Pyrenochaeta, Cylindrocarpon and Tomentella. Dominant fungi at the decaying period were Chaetomium, Chaetomium, Corynascella, Un--s-Sordariales sp, Un--s-Tremellomycetes sp, Aspergillus, Retroconis, Un--s-Chaetomiaceae sp, Un--s-Fungi sp, Penicillium, Pulvinula and Pyrenochaeta. The abundance of Chaetomium at the decaying period was significantly higher than that of mature period, squaring period, vigorous growing period and resettling stage. Dominant fungi at the squaring period were Un--s-Tremellomycetes sp, Aspergillus, Corynascella, Chaetomium, Retroconis, Pulvinula, Penicillium, Un--s-Chaetomiaceae sp, Chrysosporium, Pyrenochaeta and Un--s-Fungi sp. Compared with fungi in rhizosphere soil at other periods, at the resettling stage, the proportion of Aspergillus increased, while the proportions of Chaetomium and Un--s-Chaomietaceae 
sp decreased. At the vigorous growing period, the proportion of Mortierella increased, while the proportions of Un--s-Sordariales sp and Un--s-Chaetomiaceae sp decreased. At the mature period, the proportion of Un--s-Fungi sp increased, while the proportions of Un--s-Tremellomycetes sp and Un--s-Sordariales sp decreased. At the decaying period, the proportion of Chaetomium increased, while the proportions of Aspergillus and Retroconis decreased. At the squaring period, the proportion of Un--s-Tremellomycetes sp increased, while the proportions of Chaetomium and Retroconis decreased.

\section{Acknowledgements}

This paper is supported by National Natural Science Foundation of China (41361056, 31460491, 31660426); Joint Special Fundamental Research of Local Universities in Yunnan Province (2017FH001-041, 2017FH001-035, 2017FH001-005); Talent Introduction Program of Kunming University (YJL14005); Key Disciplines (Ecology) Project of Yunnan Education Department (05000511311); Open Fund Project of Key Laboratory of Characteristic Biological Resources Development and Utilization in Yunnan Province (GXKZ201716); China National Tobacco Corp Science and Technology Project (2018530000241020, 2018530000241016).

\section{References}

[1] G.S. Liu, Y.T. Wang, Y.F. Wang, et al., Tobacco Cultivation, China Agriculture Press, Beijing, 2003.

[2] Z.G. Li, L.Q. Ma, A.G. Wang, et al., Changes of microbes in rhizosphere soil of tobacco at different growth stages in western Henan Province, J. Guizhou Agricultural Sciences. 44 (2016).

[3] X.H. Wu, W.X. Liu, Soil Microbial Ecological Engineering, Chemical Industry Press, Beijing, 2012.

[4] Y.Z. Fan, Changes of rhizosphere microorganisms of Trifolium repens at different growth stages, J. Jiangsu Agricultural Sciences. 5 (2010).

[5] J.B. Sun, L.P. Qu, W.B. Li, et al., Changes of bacterial communities in rhizosphere soil of banana at different growth stages, J. Chinese Journal of Tropical Crop. 27 (2016).

[6] Q.H. Wu, Y.T. Ma, G.Z. Wang, et al., Study of dynamic characteristics of soil microorganism in rhizosphere soils of Aconitum carmichaeli of different trophophase, J. Chengdu Traditional Chinese Medicine. 26 (2006).

[7] X.L. Liu, C. Wang, F. Wu, et al., Studies on tobacco rhizosphere microbes, J. Acta Ecologica Sinic. 26 (2006).

[8] A.M. Cortes-Barco, P.H. Goodwin, T. Hsiang, et al., Comparison of induced resistance activated by benzothiadiazole, (2R, 3R) - butanediol and an isoparaffin mixture against anthracnose of Nicotiana benthamiana, J. Plant Pathology. 59 (2010).

[9] Y.H. Yang, D.M. Chen, Y. Jin, et al., Effects of different fertilizers on functional diversities of microbial flora in rhizospheric soil of monoculture tobacco, J. Acta Agronomica Sinica. 37 (2011).

[10] C. Wang, F. Wu, X.L. Liu, et al., Tobacco rhizosphere microorganism in different fertility of soil, J. Chinese Tobacco Science. 26 (2005). 